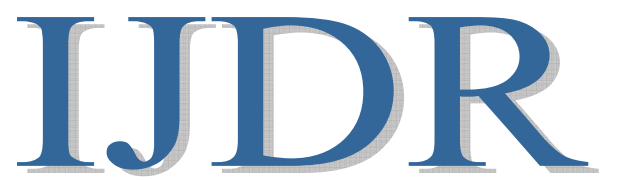

International Journal of Development Research

Vol. 10, Issue, 09, pp. 40074-40079, September, 2020

https://doi.org/10.37118/ijdr.197421.09.2020

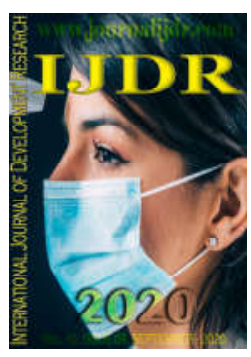

\title{
FUNCTIONAL BANDAGE ON BIOMECHANICAL PARAMETERS IN THE KNEE
}

\section{Ramiro Marques Inchauspe* and Pablo Morales Barbian}

\author{
Federal University of Health Sciences of Rio Grande do Sul, Porto Alegre, Brazil
}

\section{ARTICLE INFO}

\section{Article History:}

Received 22 $2^{\text {nd }}$ June 2020

Received in revised form

$04^{\text {th }}$ July 2020

Accepted $19^{\text {th }}$ August 2020

Published online $29^{\text {th }}$ September 2020

\section{Key Words:}

Functional Elastic Bandage,

Gait Analysis, Muscle Activity,

Fascial Chains, Kinesiology.

*Corresponding author:

Ramiro Marques Inchauspe

\begin{abstract}
Background: Postural changes in the lower limb influencebiomechanically and functionally the other joint complexes, which may be related to myofascial dysfunctions. The functional elastic bandages seem to promote muscle activation that may go against the valgus pattern of the knee. Objective: To evaluate the acute response of the use of elastic bandages applied in the direction of the line of action of both fascial chains, valgus or varus, during a gait cycle. Methods: A descriptive case study was carried out with a young adult volunteer with genuine valgus and pronated feet. Electromyographic data were collected from the Middle Gluteal, Tensor Fascia Lata, Vasto Lateral and Vasto Medial muscles during gait at two speeds and two techniques for applying different functional bandages. The order of speeds and techniques for applying functional bandages was randomized by simple drawing. For the kinematic capture of gait, support and balance cycles, a Cinemetry system with 6 infrared cameras was used. The Electromyographic signal was processed to obtain the maximum voluntary contraction. Results: The results were divided into a sign of inhibited muscle activation or with greater activation during the execution of the movement. There was greater activation of the middle gluteal muscle during the support phase in gait with the use of varus banding and greater activation of the vastus during the same phase with the use of valgus bandage. Conclusion: In this case study, different strategies for applying functional bandages impacted different neuromuscular responses during gait.
\end{abstract}

Copyright (C) 2020, Ramiro Marques Inchauspe and Pablo Morales Barbian. This is an open access article distributed under the Creative Commons Attribution License, which permits unrestricted use, distribution, and reproduction in any medium, provided the original work is properly cited.

Citation: Ramiro Marques Inchauspe and Pablo Morales Barbian. 2020. "Functional bandage on biomechanical parameters in the knee", International Journal of Development Research, 10, (09), 40074-40079.

\section{INTRODUCTION}

Postural dysfunctions and misalignments of the lower limb (LL) can lead to biomechanical changes and influence the functionality of important structures, leading to osteochondral lesions (Czaprowski, 2018). The stability of the patellofemoral joint is a result of a complex interaction between several factors, including the joint geometry of the patella and femur, the magnitude and quadriceps muscle lines-of-action, the stiffening of the retinacula and the rotation of the tibia (Loudon, 2016). This process results in excessive pronation of the foot, which, during the heel support phase during gait, increases eversion and, consequently, medial rotation of the talus and functional bandages and neuromuscular activity in the genu valgus and tibia gait.To reach full knee extension, the femur will perform a medial rotation (McConnell, 1996). The weakning of the hip muscles, such as the middle gluteus (MG), causes the fall of the contralateral pelvis, increase internal rotation and adduction of the ipsilateral femur (dynamic valgus) during functional movements, such as walking.
This process indicates that the dynamic valgus is related to strength, coordination, skill, anatomical alignment and underlying arthrocinmatic function.The $\mathrm{MG}$ is an important abductor of the hip, the greater the hip flexion, the greater the activation of this muscle. The strengthening of the $\mathrm{MG}$ is described in the treatment of patellofemoral dysfunction and shows a decrease in the excess of dynamic valgus (Salsich, 2002). The balance in muscle activity between the vastus lateralis (VL), vastus medialis obliquus (VMO) and vastus medial (VM) is essential for an adequate relationship between the patella and the femoral trochlea. If there is an imbalance in the activity of these two muscles due to dysfunction of the VMO, an inappropriate lateral path of the patella may occur (Steinkamp, 1993).Aiming a treatment, in 1984 Jenny McConnel presented a concept of rigid functional bandaging, initially used to correct abnormal patellar positions in athletes with femoral patellar pain syndrome ${ }^{6}$. Another method proposed by Kenzo Kaze in 1990 is the application of a hypoallergenic cotton adhesive tape that can be deformed up to $140 \%$ of its original size. It has the characteristic of being 
similar to human skin and has numerous mechanisms of action, such as: biomechanical, exteroceptive, circulatory, etc. The functional elastic bandage promoted the skin lifting, increasing blood and lymphatic circulation in the subdermal (Williams, 2012).The muscles, regardless of their individual function, also influence the body continuity that performs the same function in the fascial network (Myers, 1997). These lines follow the twists and wefts of the connective tissue, forming "meridian" strokes of the myofascia. Stability, deformation, tension, fixation, elasticity, and postural compensations are attributed to these lines.Following this concept, the spiral line connects the arch of the foot with the pelvic angle and helps to establish the knee path during gait. When an imbalance occurs, the spiral line creates, compensates and maintains curves, rotations and lateral displacements of the body. The spiral line passes over the anterosuperior iliac spine (ASIS), being considered as a transition station in the myofascial continuity theory, in order to observe the different mechanical tractions from this point ${ }^{9}$. Functional lines are less involved with maintaining upright posture than others previously mentioned. They involve muscles used for day-to-day activities that have a minimal opportunity to maintain posture, but act on dynamic postural stabilization (5). In a recent systematic review (Wilke, 2016), it was demonstrated that most of the anatomical fascial chains were proven through anatomical studies in cadavers. Except for the frontal superficial chain, the spiral chain (valgus because it involves the line of action of the anterior tibial, ileotibial tract and the Tensor of the Fascia Lata (TFL)) and the deep anterior chain (varus for coinciding with the lines of action of the posterior tibial muscles, adductor muscles and external hip rotators) presented moderate consistency.The elastic or functional bandages seem to act on the skin and possibly on the superficial fascias of the body and no research has been found that investigated the neuromuscular response of muscles involved with valgus knee and with the pronation of the standing in functional situations. Considering the above lack, this paper has the aim to investigate the acute response of the use of elastic bandages applied in the direction of the action line of both fascial chains, valgus or varus, during a gait cycle.

\section{METHODS}

The present research project, registered under number 20013 was approved by the Research Ethics Committee of the Federal University of Rio Grande do Sul and developed following the guidelines of Resolution No. 196/96 of the National Health Council. The data collections were carried out at the Exercise Research Laboratory of the Physical Education School of the Federal University of Rio Grande do Sul.

\section{SAMPLES}

This is a case study, with an exploratory-descriptive profile, whose sample was non-probabilistic and selected for convenience. A woman, aged 21 years, with a height of 163 $\mathrm{cm}$ and a body mass of $58 \mathrm{~kg}$ participated in this study. The volunteer, although she did not have a history of osteomioarticular diseases that could interfere with the results, had a genuine bilateral valgus bandage, neuromuscular activity in the genu valgus and gait with both feet pronated, whose postural alteration was confirmed by physical therapy evaluation. The participant read and signed the consent form agreeing with her participation in the study.

\section{EQUIPMENTS}

For the acquisition of electromyographic data (EMG) during the task of walking on a treadmill, a four-channel Miotool 400 electromyograph (Miotec ${ }^{\circledR}$ EquipamentosBiomédicos, Porto Alegre, Brazil) was used, with a sampling frequency of 2000 $\mathrm{Hz}$ per channel, gain range of 200 to 1000 times and common rejection mode greater than $126 \mathrm{Db}$. Surface adhesive electrodes of bipolar configuration model Mini Medi-trace 100 (Kendall ${ }^{\circledR}$, Canada) with $10 \mathrm{~mm}$ conductive area radius and $15 \mathrm{~mm}$ total radius were used. To evaluate the displacement of the lower limb during gait, a model 10200 ATL treadmill, IMBRAMED (Porto Alegre, Brazil) was used. For the kinematic capture of gait cycles, a Cinemetry system with 6 infrared cameras was used. The obtainment of the threedimensional angular kinematic parameters of the gait was performed by means of a movement analysis system (Vicon ${ }^{\circledR}$ Motion Systems). The images were captured by seven $100 \mathrm{HZ}$ infrared cameras, which record the position and movements of the reflective markers fixed at anatomical reference points and the NEXUS software (Vicon ${ }^{\circledR}$ Motion Systems, model 624, Oxford, United Kingdom) identifies the spatial coordinates of the markers.

\section{DATA COLLECTION PROCEDURES}

For the collection of electromyographic data, prior to placing the electrodes, the skin was prepared with trichotomy, abrasion and asepsis with $70 \%$ alcohol. Pairs of surface electrodes were placed in the muscles of the right lower limb in the VM, VL, MG and TFL. Skin preparation and electrode placement followed the recommendations of the Surface Electromyography for the Non-Invasive Assesment of Muscles (SENIAM). The reference electrode was positioned on the face of the lateral malleolus of the opposite lower limb. For comparison purposes, the signals from the monitored muscles were normalized from the tests of Maximum Voluntary Contractions (MVCs), lasting five seconds. For the execution of MVCs, individuals were verbally encouraged.

\section{Functional Elastic Bandages}

The application of elastic bandage was performed based on the technique of "mechanical correction". The stresses for this type of correction are performed from $50 \%$ of the tape stretch (Kenzo, 2003). To measure the stretch of $50 \%$, a tape measure was used during the application of the bandage. A single trained person applied the bandages to the volunteer. The application of bandages consisted of two distinct moments and separated by a week apart. Two modes of application of the bandages were used, namely: Techniques to favor fascial chains opposite to the genuine valgus, called varusbandage; and technique favoring fascial chains of ganu valgus, called valgusbandage. The technique applied was drawn by the researcher and the result of the draw was hidden from the volunteer. The technique adopted for the varus Bandage sought: The external rotation of the hip joint, being applied over the Gluteus Maximo muscle; The internal rotation of the tibia in relation to the femur, being applied on the goose leg; Supination of the foot, being applied to the plantar region of the center of the longitudinal arch of the foot, passing over the navicular bone and following the line of action of the posterior tibial muscle over the anterior Tibial muscle. 


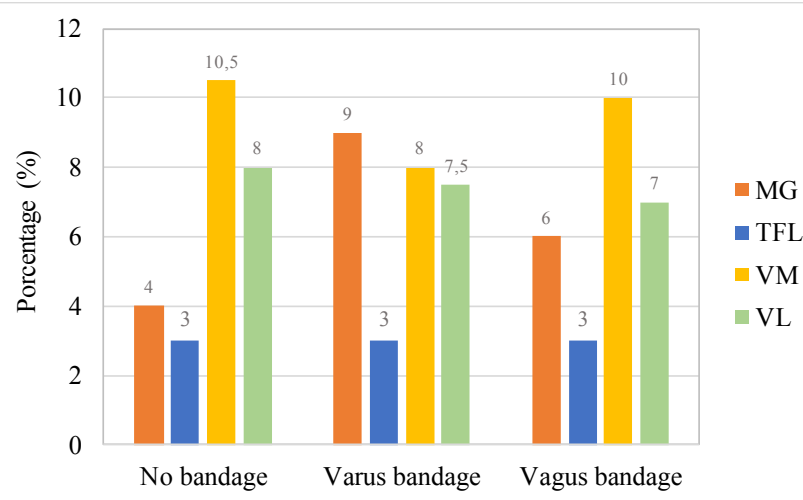

Figure 1. Percentage of muscle activation normalized by MVC during the support phase of walking at a speed of $4 \mathrm{~km} / \mathrm{h}$

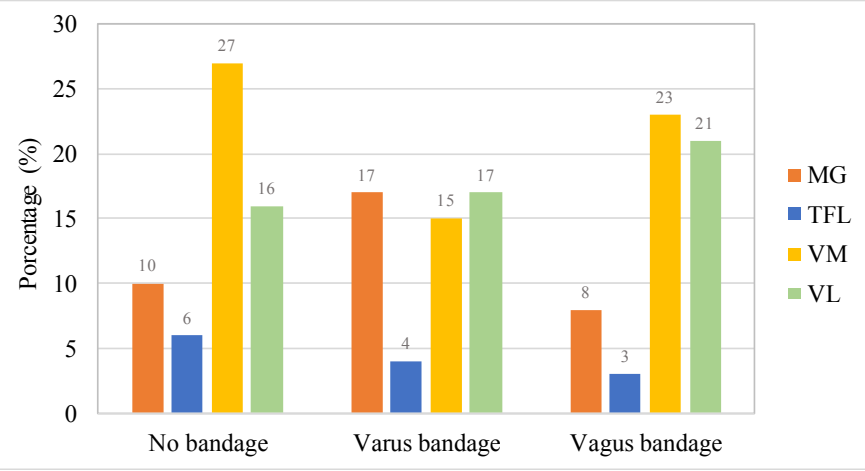

Figure 2. Percentage of muscle activation normalized by MVC during the balance phase of walking at a speed of $4 \mathrm{~km} / \mathrm{h}$

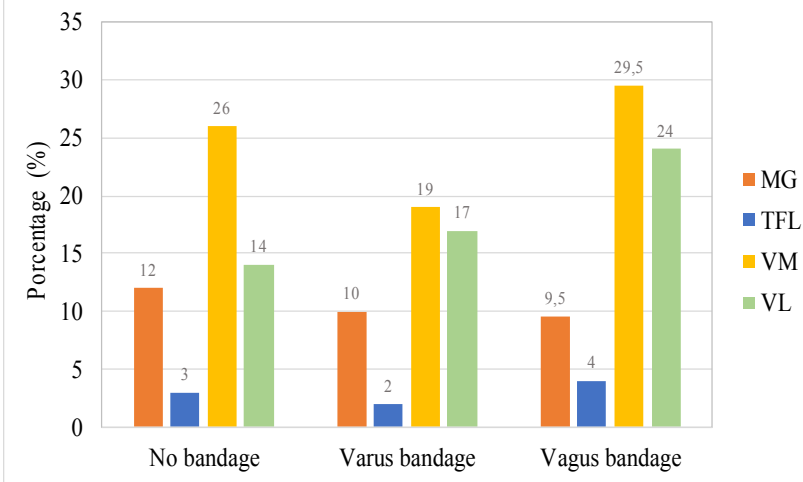

Figure 3. Percentage of muscle activation normalized by MVC during the support phase of walking at a speed of $5 \mathrm{~km} / \mathrm{h}$.

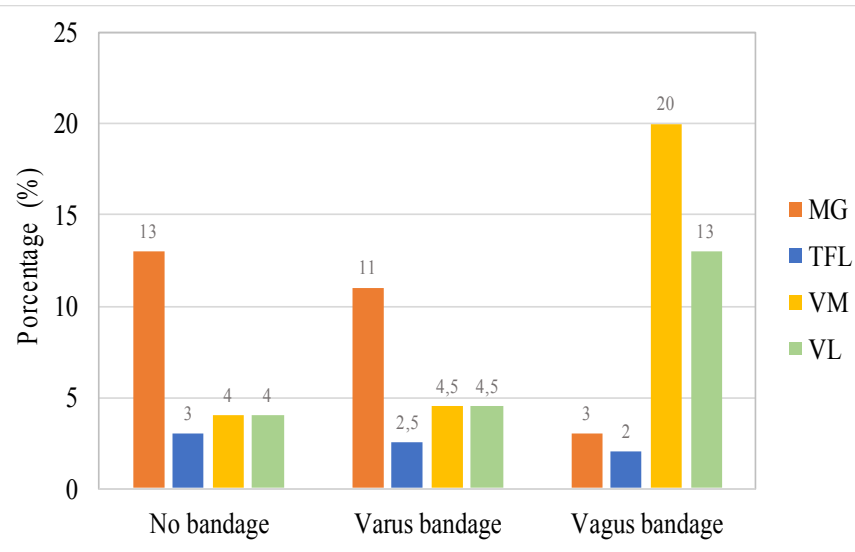

Figure 4. Percentage of muscle activation normalized by MVC during the balance phase of walking at a speed of $5 \mathrm{~km} / \mathrm{h}$.
The application technique of the valgusbandage sought: The internal rotation of the hip, applied on the TFL muscle; external rotation of the tibia under the femur, applied over the TIT in the knee; and pronation of the foot was "encouraged" by applying a bandage to the plantar surface of the foot, passing over the base of the 5th metatarsal and following the line of action of the fibular muscles. In both situations, the techniques were performed with the volunteer in the supine position and the hip, knee and ankle joints remained in a neutral position.

\section{Procedures}

The volunteer was instructed to walk in a straight line on the treadmill without inclination, in two situations, for a period of 30 seconds. Two walking speeds were determined: Fixed gait analysis, with a speed of $4 \mathrm{~km} / \mathrm{h}$; and Self-selected gait analysis, with a speed of $5 \mathrm{~km} / \mathrm{h}$. The selection order of walking was based on functional bandages and neuromuscular activity in the genuine valgus and gait previously drawn and informed to the volunteer.During the march, the electromyographic activity of the muscles captured the VM, $\mathrm{VL}, \mathrm{MG}$ and TFL. Image capture is carried out by the camera system to analyze the phases of support and balance during the march. After the first analysis, performed without bandaging, the researcher selected the bandage technique to be applied, and a new collection was carried out. Between the collection of gait analysis and electromyographic activity of the two techniques, there was an interval of one week for the reaccommodation of the involved fascia tissues.

\section{Data Analysis}

The files were exported to Biomec SAD32 (developed by the Laboratory of Mechanical Measurements, UFRGS, Brazil). An Ideal digital filter with a cutoff frequency between 10 and 500 $\mathrm{Hz}$ and a second order Butterworth digital filter with a frequency of cut between 50 and $500 \mathrm{~Hz}$. Then, the phase of interest was cut and the root mean square (RMS) was obtained. The RMS values were obtained from all muscles and normalized by the MVCs values.Since it is a case study, descriptive statistics were used, with values normalized by the Maximum Isometric Voluntary Contraction of the studied muscles.

\section{RESULTS}

The results found in this study, since it is only one participant, cannot be reproduced to describe the population in which the sample is inserted. The following results are presented as a percentage in relation to the normalization acquired from muscle activity in Maximum Voluntary Contraction (MVCs). Analyzing the swing phase of the gait at a fixed speed of 4 $\mathrm{km} / \mathrm{h}$ with the application of the varus bandage, we can observe a greater activation of the GM muscle $(5.858 \%)$ and an inhibition of the other muscles: TFL (-0.083\%), VM ($2.873 \%)$ and VL $(-0.892 \%)$ in relation to walking at the same speed without bandaging. When comparing the results obtained from walking at $4 \mathrm{~km} / \mathrm{h}$ with the varus bandage in relation to walking at the same speed without badness, we observed again an increase in the activity of the MG muscle (2.961\%), however, more discreet, and an inhibition of the other muscles: TFL (0.431\%); VMO (-0.751\%) and VL ($1.169 \%)$. The results obtained during the $4 \mathrm{~km} / \mathrm{h}$ gait phase with varus banding again showed a greater activation of $\mathrm{MG}$ 
$(6.483 \%)$ and an inhibition of the TFL $(-3.243 \%)$, VL $(0.541 \%)$, and mainly VM muscles $(-12.237 \%)$ in relation to walking without bandaging. On the other hand, the results of walking at the same speed with the valgus bandage in relation to walking without bandaging show a decrease in the MG ($3.019 \%)$, TFL (3.245\%) and VMO (-3.738\%) muscles, and an activation in the VL $(4.614 \%)$. The data of the balance phase of the self-selected gait $(5 \mathrm{~km} / \mathrm{h})$ with the varus bandage point to the inhibition of the MG (-1.417\%), TFL (-0.676\%), VM ($6.355 \%)$ and VL (6.964\%) muscles. A greater activation of TFL (1.098\%) and VM (4.089\%), and a decrease in the activation of MG (-1.890\%) and VL $(0.787 \%)$, are obtained from the confrontation of data between the gears of the same speed with valgus bandaging and without bandaging. During the self-selected gait support phase $(5 \mathrm{~km} / \mathrm{h})$ with varus banding in relation to the same gait phase without bandaging, there was a decrease in the neuromuscular activity of all the analyzed muscles: MG (-2.271\%); TFL (-0.622\%); VM $(0.843 \%)$ and VL $(-0.047 \%)$.Analyzing the phase of support of the Self-selected gait with valgus banding in relation to the one without banding, there was an expressive activation of the vastus, VM (15.867\%) and VL (8.923\%), while the MG ($10.376 \%)$ and TFL (-1.465\%) decreased activity. Below are the tables with all the results found, as well as the histograms representing the collected data.

\section{DISCUSSION}

The volunteer showed different neuromuscular activity patterns when walking at the self-selected speed $(5 \mathrm{~km} / \mathrm{h})$ and at the speed determined by the researchers $(4 \mathrm{~km} / \mathrm{h})$.It is noticed that, even without the application of bandaging, when the volunteer walked at the self-selected speed, during the support phase, there was less activity of the muscles representing the quadriceps (VM), which worked with a percentage less than $5 \%$ of the MVCs, while at the speed determined by the researchers, both muscles reached activities above $15 \%$, including VMO above $20 \%$. In addition, even in the support phase, the TFL and GM muscles showed a slight difference. At the self-selected speed, there was less activation of the TFL and greater activation of the MG. At the speed determined by the researchers, the volunteer had a greater activation of the TFL.Tartaruga et al. (Tartaruga, 2012)report that the efficiency standards during the run either indicate an adaptation in the chosen gait through training (biomechanical adaptation) or a successful energy optimization process. Studies indicate that the synergistic relationship between TFL and GM has an important correlation with the angle of rotation of the hip and, consequently, with the Q angle of the knee. The correlation between hip adduction and valgus knee provides empirical evidence for the collapse of the knee medial complex. Hollman et al. (Hollman, 2009) postulated that excessive internal rotation and secondary to inadequate hip muscle function can result in abnormal mechanics, which leads to injury. The lower gait speed may have influenced an increase in TFL activity, which would lead to greater stress on the iliotibial tract, consequently transferring its forces to the lateral retinaculum of the patella, increasing its lateralization and impact on the condyle lateral of the femur. Powers et al. ${ }^{13}$, describes that the relationship between hip adduction and valgus knee can increase the resulting lateral forces active on the patella, which can increase the lateral patellar traction and the lateral joint pressure of the patellofemoral.On the other hand, still observing the gait without bandaging, in the swing phase (when there must be a predominance of the action of the hamstrings; 4), at the self-selected speed there was a greater activity of the vast ones. The quadriceps function to cause extension in the final phase of the swing. There is the possibility that, at the self-selected speed, the individual may have accelerated the knee extension more in the final phase of the swing.During the balance phase, the anterior portion of the MG can act as a hip flexor and also work in synergy with the adductors, controlling the hip rotations and even the movements in the frontal plane, adduction and abduction. It is noticed that in the self-selected speed, with both banding techniques, the MG presented greater activity than in the speed determined by the researchers. The neuromuscular activity of the muscles involved in the genuine valgus and in the opposite of this same postural alteration during the speed determined by the researchers, it is noticed that in the support phase, using the bandage that corroborates the valgus pattern, there was a greater activity of the VM. According to Fredericsonet al. ${ }^{14}$, the weakness of MG leads to a decrease in the control of abduction and external rotation. Subsequently, a faster gait will lead to an abduction and internal rotation of the hip, increasing the knee valgus. The possible increase in the electromyographic activity of the VMO may be due to the fact that a reduction in the activity in relation to the bandage that fights the valgus. The MG had a significant action in relation to the bandage that corroborates the valgus, so the increase in the action of the MG may have reduced the demand on the VM to stabilize the medial patellar because the external rotation of the femur by itself would reduce the genu valgus. On the other hand, using the valgus bandage, the low activity of the MG in the support phase may have demanded greater action of the VM (because the VM had a greater demand for stabilization). The VM had a small increase in activity with the use of the varus bandage, while the VL had less activity. Since the valgus bandage reduced the activity of the $\mathrm{MG}$ in the support phase, it demanded a greater activity of the VMO, and a possible inhibition of the VL, through reciprocal inhibition.Femoral adduction that puts the knee into an enlargement of the valgus may be associated with insufficient recruitment of the MG muscle during the balance phase, counterbalancing external demands to maintain optimal hip alignment.

The results also suggest that MG activation may have a greater association with knee alignment than just hip muscle strength in the frontal plane in women (Hollman, 2009).Varus banding seems to significantly increase the activity of the GM, requiring less action by the $\mathrm{VM}$ and the oblique $\mathrm{VM}$ for this instability, and allowing the VM to increase its activity. These data are very important considering that most knee injuries occur during the support phase during gait and running. When there is a strong impact and protrusion of the patella and when the opposition of the internal rotations of the femur and the external tibia overload the patellofemoral, the tibiofemoral, through the stress of the iliotibial tract that transfers its forces through the lateral retinaculum of the patella, increasing the impaction of the condyle and increasing the tilt and bad congruence of the patella in the intercondylar trochanea of the femur.On the other hand, in the balance phase, we know that the synergy between the MG and the TFL is important to control the hip rotation. If the hip performs a very significant internal rotation in the balance phase and lands with this hip internally rotated, this will exacerbate the valgus and its possible associated injuries.It is noticed that using the varus bandage there is also a greater activity of the $\mathrm{MG}$ in the balance and a lesser relative activity of the TFL, indicating that even in the phase of balance there is a better control of the hip 
and better synergistic interrelation of these two muscles while the hip flect, probably decreasing the rotational factor of this joint. The vastus had no apparent difference during the balancing phase, when considering arbitrary speed when comparing the two bandages.Speed is responsible for the greater amount of biomechanical processes that induce pathologies and injuries, for the increased load on the articular elements of the lower limb and, consequently, for an increase in the shear of the patellofemoral and tibiofemoral components. It is noticed that in the support phase, the $\mathrm{MG}$ speed had an increase in activity when compared to the bandage that accentuates the valgus. Once again, it can be seen that the increase in MG activity in relation to TFL seems to be positive in this control of the patellofemoral, knee and hip joint. While analyzing the self-selected speed, it is clear that in the support phase, considered by the researchers to be the most important, as it is responsible for the largest amount of biomechanical processes that induce pathologies and injuries, by increasing the load on the limb's joint elements lower and consequently the increased shear of the patellofemoral and tibiofemoral components, it is noticed, once again, that at this speed the GM had an increase in activity when compared to the bandage that accentuates the valgus. Once more noticed that the increase in the activity of the GM in relation to the TFL seems to be positive in this control of the patellofemoral, knee and hip joint.

The use of the varus bandage had a small increase in TFL, however, even with this increase the MG/TFL ratio remained positive for MG. With regard to the vastus areas, there was less activity with the use of the varus bandage, and greater activity with the use of the valgus bandage. Perhaps the instability or the mechanical stress created by the bandage itself, or the somatosensory signaling coming from the fasciae, may have triggered an increase in the excitement of these vastus regions trying to protect the patella. Another case, on the opposite may happened: the good stability of the patella, generated by the varus bandage, it may have reduced the demand for activity in the vast areas during the support phase. On the other hand, during the balance phase, the MG once again had an increase in activity with the varus bandage when compared to the use of the valgus bandage. With respect to the vast areas, there does not seem to be much difference, with the exception of VM, which, with the use of valgus bandage, seems to have increased its demand.Afferent proprioceptors in fasciae can mediate afferences that trigger motor interactions, or motor responses arising from the stress of shortening and stretching. What we understand is that when the fascia is distended, probably the proprioceptors from this stretched fascia can send signals via the medulla, brain stem, or even cortex, or various structures of the central nervous system and contribute to the correction of this postural alteration. The discovery of contractile cells, end of free innervations and mechanoceptors suggests that the fascia plays a proprioceptive and mechanically active role. The fascia can transmit tension and in view of its proprioceptive and nociceptive function, the existence of meridians can be responsible for disorders and pain irradiation to remote anatomical structures (Wilke, 2016).The hip provides the greatest contribution to the moment of total support during the initial phase of support. The compensation strategy for loss of knee stability relies on good hip stability (Houck, 2005).According to Parreiraet al., (2014) functional elastic bandages are widely used in clinical practice, but without effectiveness. However, situations in which functional bandages were performed based on the fascial lines proposed by Myers $^{8}$ were not analyzed.During soft tissue manipulation, such as yoga postures and smooth active stretches, Golgi receptors are stimulated, which results in a lower firing rate of the specific Alpha motor neuron, which translates into a reduction in related tissue tone ${ }^{17}$.

\section{Conclusion}

From this paper, we conclude that, for this subject, the application of the varus bandageas corrective technique for valgus and pronated foot, seems to rebalance the synergistic actions of the muscles involved in this postural alteration during gait. Further studies are suggested using this resource based on the classification of Myers chain.

\section{Funding}

This research did not receive any specific grant from funding agencies in the public, commercial, ornot-for-profit sectors.

\section{REFERENCES}

Czaprowski D, Stoliński L, Tyrakowski M, Kozinoga M, Kotwicki T. Non-structural misalignments of body posture in the sagittal plane. Scoliosis Spinal Disord. 2018; 13: 6. https://doi.org/10.1186/s13013-018-0151-5.

Fredericson M, Cookingham CL, Chaudhari AM, Dowdell BC, Oestreicher N, Sahrmann SA. Hip abductor weakness in distance runners with iliotibial band syndrome. Clin J Sport Med. 2000;10(3):169-75. https://doi.org/10.1097/00042752-200007000-00004.

Hollman JH, Ginos BE, Kozuchowski J, Vaughn AS, Krause DA, Youdas JW. Relationships between knee valgus, hipmuscle strength, and hip-muscle recruitment during a single-limb stepdown. J Sport Rehabil. 2009;18(1):104117. https://doi.org/10.1123/jsr.18.1.104

Houck JR, Duncan A, De Haven KE. Knee and hip angle and moment adaptations during cutting tasks in subjects with anterior cruciate ligament deficiency classified as noncopers. J Orthop Sports Phys Ther. 2005;35(8):53140. https://doi.org/10.2519/jospt.2005.35.8.531.

Loudon JK. Biomechanics and pathomechanics of the patellofemoral joint. Int J Sports Phys Ther. 2016 Dec; 11(6): 820-830.

McConnell J. Management of patellofemoral problems..pdf. Manual Therapy. 1996;60-6. https://doi.org/1 0.1054/ math.1996.0251.

McConnell J. Management of patellofemoral problems..pdf. Manual Therapy. 1996;60-6. https://doi.org/10.1 054/math.1996.0251.

Myers TW. Kinesthetic dystonia: what bodywork can offer a new physical education. J Bodyw Mov Ther. 1998;2(2):101-114. https://doi.org/10.1016/S1360-8592 (98)80031-4.

Myers TW. The anatomy Trains. J Bodyw Mov Ther. 1997;1:91-101.

Parreira PDCS, Costa LDCM, Hespanhol Junior LC, Lopes AD, Costa LOP. Current evidence does not support the use of Kinesio Taping in clinical practice: A systematic review. $J$ Physiother [Internet]. Korea Institute of Oriental Medicine; 2014;60(1):31-9. https://doi.org/1 0.1016/j.jphys.2013.12.008.

Powers CM, Landel R, Perry J. Timing and intensity of vastus muscle activity during functional activities in subjects with and without patellofemoral pain. Phys Ther. 
1996;76(9):946-55;

discussion

https://doi.org/10.1093/ptj/76.9.946

Salsich GB, Brechter JH, Farwell D, Powers CM. The effects of patellar taping on knee kinetics, kinematics, and vastus lateralis muscle activity during stair ambulation in individuals with patellofemoral pain. J Orthop Sports Phys Ther. 2002;32(1):3-10. https://doi.org/10.25 19/jospt.2002.32.1.3.

Schleip R. Fascial plasticity - A new neurobiological explanation Part 2. J Bodyw Mov Ther. 2003;7(2):104116. https://doi.org/10.1016/S1360-8592(02)00076-1.

Steinkamp LA, Dillingham MF, Markel MD, Hill JA, Kaufman KR. Biomechanical considerations in patellofemoral joint rehabilitation. Am J Sports Med. 1993;21(3):438- 44. https://doi.org/10.1177/036354 659302100319.
Tartaruga MP, Brisswalter J, Peyré-Tartaruga LA, AvilaAO, Alberton CL, Coertjens M, Cadore EL, Tiggemann CL, Silva EM, Kruel LF. The Relationship Between Running Economy and Biomechanical Variables in Distance Runners. Res Q Exerc Sport. 2012;83:3:367-375. https://doi.org/10.1080/02701367.2012.10599870.

Wilke J, Krause F, Vogt L, Banzer W. What Is EvidenceBased About Myofascial Chains: A Systematic Review. Arch Phys Med Rehabil. 2016 Mar;97(3):454-61. https://doi.org/10.1016/j.apmr.2015.07.023.

Williams S, Whatman C, Hume PA., Sheerin K. Kinesio taping in treatment and prevention of sports injuries: A meta-analysis of the evidence for its effectiveness. Sport Med. 2012;42(2):153-64. https://doi.org/10.2165/1 159496 0-000000000-00000 